\title{
MORBIMORTALIDAD POR CÁNCER DE RECTO OPERADO CON TÉCNICA DE MILES EN EL HOSPITAL ESSALUD DE ICA, PERÚ 1993-2003
}

\author{
Félix Lem-Arce ${ }^{1,2, a, b .}$, Ariel Guerra-Alvarez ${ }^{1, c .}$ \\ 1. Facultad de Medicina. Universidad Nacional San Luis Gonzaga. Ica. Perú. \\ 2. Hospital Félix Torrealva Gutiérrez, EsSalud. Ica, Perú. \\ a. Cirujano Oncólogo. b. Magister en Medicina. c. Cirujano General.
}

\section{RESUMEN}

El cáncer de colon y recto, es una enfermedad frecuente, cuyo tratamiento indicado es la cirugía con carácter de curativa. La operación aceptada a nivel mundial es la resección abdominal-perineal (Miles). Se han operado 29 pacientes, 15 hombres y 14 mujeres, con edades entre 43 y 84 años. No hemos tenido ninguna muerte post operatoria y la tasa de complicaciones es de $20,6 \%$ (6/29) infecciones de la herida operatoria, la estancia hospitalaria fue de 9 a 11 días, el tiempo operatorio promedio de 3 a 4 horas, 24 pacientes sobreviven mas de 5 años $(82,7 \%)$ el tipo histológico mas frecuente fue adenocarcinoma concordante con otros estudios publicados. (Rev. méd. panacea 2011; 1:14-16).

Palabras clave: Cáncer de recto, Resección abdominal - perineal. Morbimortalidad (fuente: DeCS BIREME)

\section{MORBIMORTALITY BY RECTAL CANCER OPERATED WITH TECHNIQUE OF MILES ESSALUD HOSPITAL IN ICA , 1993-2003.}

\begin{abstract}
The colon and rectum cancer is a common disease, their treatment is indicated through the surgery with curative character. The surgery accepted globally is the perineal - abdominal resection (Miles). 29 patients have been operated, 15 men and 14 women, their ages ranged between 43 and 84 . There were not any death after surgery but the rate of complications was $20.6 \%(6 / 29)$ infections in surgical wounds. The stay in hospital was 9 to 11 days. The average time of these operations was 3 to 4 hours, 24 patients survived more than 5 years $(82.7 \%)$. The most frequent histological type was adenocarcinoma which is concordant with another published studies. (Rev. méd. panacea 2011; 1:14-16).
\end{abstract}

Key words: Rectal Cancer. Miles operation. Morbimortality (source: MeSH NLM).

\section{INTRODUCCIÓN}

El cáncer de colon y recto es la neoplasia que ocupa el sexto lugar en el Perú. Su frecuencia aumenta con la edad avanzada y su tratamiento de elección es la cirugía. Para el tratamiento específico del cáncer de recto, existen diferentes técnicas quirúrgicas $(1,2)$. Una de ellas es la Resección Abomino perineal u operación de Miles, y es la que hemos empleado en nuestro hospital $(3,4,5)$.

\section{PACIENTES Y MÉTODOS}

Es un estudio observacional, descriptivo, retrospectivo y analítico. Se operaron 29 pacientes, por cáncer colon rectal, en el hospital de nivel III Félix Torrealva Gutiérrez,
EsSalud, Ica, entre los años 1993-2003 que comprende el universo total de pacientes operados con esta técnica.

Se usó como instrumento una ficha de recolección de datos.

El análisis estadístico evaluó la distribución de frecuencias porcentuales de las variables.

\section{RESULTADOS}

La edad media de los pacientes fue de 74 años, edad mínima 43 máxima 84 , el mayor número de pacientes estaba entre los 71 y 77 años (48,27\%), sin mayor diferencias en cuanto al sexo (15 hombres y 14 mujeres), 17 procedían de la provincia de lca y el resto de Pisco y Chincha, nueve eran hipertensos y cuatro eran diabéticos 
Todos los pacientes recibieron preparación mecánica del colon más antibiótico, además en el $93 \%$ de los pacientes se les realizo una biopsia previa y cuyos resultados se muestran en la Figura 1.

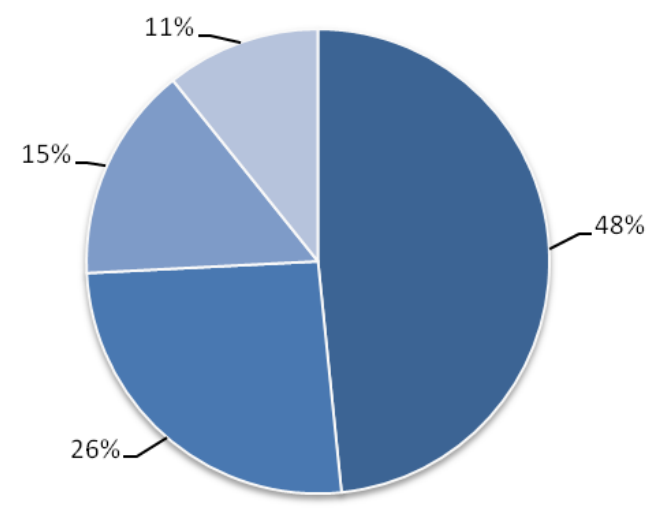

- Adenocarcinoma medianamente diferenciado

- Adenocarcinoma infiltrante medianamente diferenciado

- Adenocarcinoma bien diferenciado

- Carcinoma epidermoide medianamente diferenciado

Figura 1. Anatomía Patológica del tumor

Según la clasificación post operatorio de los estadios TNM (Tumor, Nudo Linfático, Metástasis) de la AJCC (American Joint Committee on Cancer) la mayoría de pacientes se encontraba en estadio III B (48,3\%), el margen anal del tumor más frecuente fue de 5 a $6 \mathrm{~cm}(62,1 \%)$, el tiempo operatorio en el $55,2 \%$ de los casos fue de tres a cuatro horas, en nueve pacientes se realizo otro tipo de operación quirúrgica asociada; sólo tres pacientes presentaron complicación intraoperatoria debido a hemorragia recibiendo transfusión sanguínea, no hubo reintervenciones, la estancia hospitalaria promedio fue de 9 a 11 días, seis pacientes $(20,64 \%)$ tuvieron complicaciones postoperatorias debido a infección de la herida operatoria. No hubo ninguna muerte intra o postoperatoria. El 82,7 de los pacientes tuvieron una sobrevivencia postoperatoria con más de 5 años (Tabla 1).

\section{DISCUSIÓN}

Se ha comparado la investigación nuestra con el de otros autores en lo que se refiere a complicaciones postoperatorias globales encontrando que nuestros resultados son mejores a otras sedes hospitalarias que estaría en relación a la localización del tumor y al tipo histológico $(8,9,10)$.

Al realizar una revisión de las tasas de mortalidad postoperatoria publicadas, encontramos reportes de altas tasas de mortalidad según Huenchullen en 1977 y Contreras en 1983 ambos con 5,5\% y 7,6\% respectivamente, en series más grandes de pacientes operados por la misma patología la tasa de mortalidad disminuye según Rosen con 1,7\% y Zaher con 1,8\%, una investigación publicada en 1999 por Fleshman y colaboradores en una serie de 152 pacientes reporta al igual que nosotros la ausencia de mortalidad postoperatoria $(1-6,11,12)$.

Cerca del $50 \%$ de los pacientes se operan en etapas avanzadas de la enfermedad (III B).

Tabla 1. Características de la morbimortalidad en pacientes con cáncer de recto. Hospital EsSalud de Ica 1993-2003.

\begin{tabular}{|c|c|c|}
\hline Características & $N=29$ & $(\%)$ \\
\hline \multicolumn{3}{|l|}{ Estadio TNM } \\
\hline II A & 6 & $(20,7)$ \\
\hline III A & 9 & $(31,0)$ \\
\hline III B & 14 & $(48,3)$ \\
\hline \multicolumn{3}{|l|}{ Margen anal del tumor } \\
\hline $0-4 \mathrm{~cm}$ & 5 & $(17,2)$ \\
\hline $5-6 \mathrm{~cm}$ & 18 & $(62,1)$ \\
\hline $7-8 \mathrm{~cm}$ & 2 & $(7,0)$ \\
\hline $9-10 \mathrm{~cm}$ & 4 & $(13,8)$ \\
\hline \multicolumn{3}{|l|}{ Horas de acto operatorio } \\
\hline 2 a 3 & 6 & $(17,2)$ \\
\hline 3 a 4 & 15 & $(55,2)$ \\
\hline 4 a 5 & 6 & $(20,7)$ \\
\hline Más de 5 & 2 & $(6,9)$ \\
\hline \multicolumn{3}{|l|}{ Otras operaciones asociadas } \\
\hline Histerectomía total + SOB & 4 & $(13,8)$ \\
\hline Colpectomía & 2 & $(6,9)$ \\
\hline Ooforectomía & 3 & $(10,3)$ \\
\hline Ninguna & 20 & $(69,0)$ \\
\hline \multicolumn{3}{|l|}{ Complicaciones intraoperatorias } \\
\hline Hemorragia & 3 & $(10,3)$ \\
\hline Ninguna & 26 & $(89,7)$ \\
\hline \multicolumn{3}{|l|}{ Complicaciones post operatorio } \\
\hline $\mathrm{Si}$ & 6 & $(20,6)$ \\
\hline No & 23 & $(79,4)$ \\
\hline \multicolumn{3}{|c|}{ Días de hospitalización post operatorio } \\
\hline 6 & 8 & $(27,6)$ \\
\hline 9 & 14 & $(48,3)$ \\
\hline 12 a 14 & 5 & $(17,2)$ \\
\hline Mayor de 15 & 2 & $(6,9)$ \\
\hline
\end{tabular}

Sobrevivencia post operatoria 
Fallecido entre 4 y 5 años post operatorio

Vivos después de 5 años

$24 \quad(82,7)$

Recibido: 12-4-11 Aprobado: 20-4-11

Financiamiento: El estudio fue autofinanciado

Conflictos de interés: El autor declara no tener conflictos de interés en la publicación de este artículo

\section{REFERENCIAS BIBLIOGRÁFICAS}

1. Huenchullen I. Amputaciòn abdòminoperineal del recto en càncer de ano y recto. Rev Chil Cir.1987; .29(4): 6570.

2. Rosen L, Veindentreimer $M$, Coller J. Et al. Mortality,morbidity, and patterns of recurrence after abdominoperineal resection for cancer of the rectum. Dis Colon Rectum. 1982; 25(10): 202-8.

3. Contreras J, Fascia $\mathbf{C}$ et al. Complicaciones de la Cirugìa de cáncer de colon y recto. Rev Chil Cir. 1983; 35- 232

4. Valenzuela D, Hermansen C, Stevens P. Et al. Tratamiento de càncer colorectal, experiencia. Rev Chil Cir. 1995; 47: 342-52.

5. Fleshman J, Wexner S, Anvail M,et al. Laparoscopic vs open abdominoperineal resection for cancer. Dis Colon Rectum. 1999; 42: 930-9.
6. Càrdenas R. Pizarro $\mathbf{P}$. Resecciòn abdominoperineal del recto, Anàlisis de la morbimortalidad operatoria. Rev Chil Cir. 2002; 1(54):21-26

7. Luna Pèrez $\mathbf{P}$, Rodríguez $\mathbf{S}$, Vega $\mathbf{J}$ et al. Morbidity and mortality following abdominoperineal resection for low rectal adeno carcinoma. Rev inv clìn 2001; 5(53):388-395.

8. Gutièrrez J. Cáncer del recto y cáncer anal. Lineamiento de diagnóstico tratamiento. La Habana: Instituto Nacional de Oncología y Radiobiología 1996; 1452: $45-89$

9. Boeing C, Squires $\mathrm{T}$, Tong $\mathrm{T}$, Montogomery $\mathrm{S}$. Cancer estatistics 1994. Cancer.1994; 44 : 7-28

10. Anderson $\mathbf{E}$, Charles $\mathbf{H}$, Matthew et al. Morbidity and mortality after abdominoperineal resection for cancer of rectum. The New England J Med.1999; 323: 12281233.

11. Seymour I, Schwartz MD. Principios de Cirugía.6a.ed.I 1995 ,26 (2) 1315-1336

12. Rothemberger D, Wong D. Abdominoperineal resection for adenocarcinoma of the low rectum. World J Surg.1992;16: 478-85

\section{Correspondencia:}

Dr. Félix Lem Arce

Correo electrónico: flemaica9@hotmail.com 